\title{
SUR ALLURES DE LA DENSITÉ DE POINCARÉ ET SES DÉRIVÉES AU VOISINAGE D'UN POINT FRONTIÈRE
}

\author{
Dédié à Monsieur le professeur Nobuyuki Suita à l'occasion \\ de son soixantième anniversaire
}

\section{SHINJI YAMASHITA}

Résumé. Soit $a$ un point frontière et isolé d'un domaine hyperbolique du plan complexe. Soit $\Pi(z)|d z|$ la métrique de Poincaré de ce domaine. Nous étudions quelques allures de $\Pi$ et ses dérivées partielles $\Pi_{z}, \Pi_{z z}$ et $\Pi_{z \bar{z}}$ au voisinage de $a$. Par exemple,

$$
\Pi_{z}(z)=O\left(|z-a|^{-2}(-\log |z-a|)^{-1}\right) \quad \text { pour } z \rightarrow a .
$$

L'exactitudes d'ordres sont démontrées. Quelques résultats sont obtenus aussi dans le cas où $a$ n'est pas isolé.

1991 Mathematics Subject Classificatıon. $\quad 30 C 99$.

1. Introduction. Soit $G$ toujours un domaine du plan complexe $C=\{|z|$ $<+\infty\}$ tel que le complément $C \backslash G$ contienne au moins deux points. Un tel domaine s'appelle hyperbolique. Alors, $G$ a la métrique de Poincaré $\Pi_{G}(z)|d z|$ avec la densité $\Pi_{G}>0$ définie par une projection holomorphe $f$ sur $G$ du disque unité ouvert $D=\{|w|<1\}$, regardé comme le revêtement universel de $G$, en notation: $f \in \operatorname{Proj}(D, G)$. Autrement dit, on définit

$$
1 / \Pi_{G}(z)=\left(1-|w|^{2}\right)\left|f^{\prime}(w)\right|,
$$

où $z=f(w), w \subseteq D$. Le côté droit est indépendant du choix particulier de $f$ et $w$, pour autant que $z=f(w)$ soit satisfaite. La dérivée $f^{\prime}$ de $f \in \operatorname{Proj}(D, G)$ ne s'annule jamais dans $D$. Soit $\partial G$ la frontière de $G$ dans $C$. Soit, de plus,

$$
\Phi_{n}(z, a)=|z-a|^{n} \log \frac{1}{|z-a|}
$$

où $a \in \boldsymbol{C}, z \in \boldsymbol{C} \backslash\{a\}$ et $n=1,2,3$. Le premier résultat est le

ThÉoRème 1. Soit $a \in \partial G$ un point isolé de $\partial G$. Alors,

Received June 5, 1992. 


$$
\lim _{z \rightarrow a} \Phi_{1}(z, a) \Pi_{G}(z)=1 / 2 \text {. }
$$

Peut-être, le théorème 1 est connu; voir, par exemple $[6$, p. 322 , Corollary 1], l'expression est un peut incomplète; nous n'insistons jamais de priorité. Je remercie la/le référée qui m'a appris le mémoire [6]. Mais, il est intéressant pour la comparaison du théorème 2 ci-dessous.

Soient, pour $z=x+i y \in C$,

$$
2(\partial / \partial z)=\partial / \partial x-i \partial / \partial y \text { et } 2(\partial / \partial \bar{z})=\partial / \partial x+\imath \partial / \partial y \text {. }
$$

Alors, l'opérateur $\Delta=4\left(\partial^{2} / \partial z \partial \bar{z}\right)$ est laplacien. Nous allons étudier quelques allures asymptotiques des dérivées, jusqu'à les secondes, de $\Pi_{G}$, allures qui sont moins précises relativement à celle pour $\Pi_{G}$ elle-même dans (1.2). Le second résultat est le

ThÉoRème 2. Soit $a \in \partial G$ un point isolé de $\partial G$. Alors, nous avons inégalités (1.3)-(1.5) suivantes:

$$
\begin{gathered}
\limsup _{z \rightarrow a} \Phi_{2}(z, a)\left|(\partial / \partial z) \Pi_{G}(z)\right| \leqq 1 \\
\limsup _{z \rightarrow a} \Phi_{3}(z, a)\left|\left(\partial^{2} / \partial z^{2}\right) \Pi_{G}(z)\right| \leqq 11 / 2 . \\
\limsup _{z \rightarrow a} \Phi_{3}(z, a)\left(\partial^{2} / \partial z \partial \bar{z}\right) \Pi_{G}(z) \leqq 2
\end{gathered}
$$

Notons que $\left(\partial^{2} / \partial \bar{z}^{2}\right) \Pi_{G}(z)=\overline{\left(\partial^{2} / \partial z^{2}\right) \Pi_{G}(z)}$ et $\left(\partial^{2} / \partial z \partial \bar{z}\right) \Pi_{G}(z) \geqq 0$. Nous ne pouvons pas affirmer l'exactitudes de trois constantes $1,11 / 2$, et 2 dans (1.3)(1.5). Mais, l'exactitudes d'ordres $\Phi_{n}(z, a)$ dans (1.3)-(1.5) se démontrent par les suivantes, où $H=D \backslash\{0\}$, disque percé ; voir la section 4 .

$$
\begin{aligned}
& \lim _{z \rightarrow 0} \Phi_{2}(z, 0)\left|(\partial / \partial z) \Pi_{H}(z)\right|=1 / 4 . \\
& \lim _{z \rightarrow 0} \Phi_{3}(z, 0)\left|\left(\partial^{2} / \partial z^{2}\right) \Pi_{H}(z)\right|=3 / 8 . \\
& \lim _{z \rightarrow 0} \Phi_{3}(z, 0)\left(\partial^{2} / \partial z \partial \bar{z}\right) \Pi_{H}(z)=1 / 8 .
\end{aligned}
$$

Dans la section 3 nous étudions des allures dans le cas où $a$ n'est pas isolé. Dans la section 5 nous étudions des allures du rayon d'univalence dans le cas où $a$ est isolé.

2. Preuve du théorème 1. Pour le disque percé :

$$
\Delta(b, S)=\{z ; 0<|z-b|<S\}, \quad b \in C, \quad S>0,
$$

nous avons l'égalité

$$
1 / \Pi_{\Delta(b, S)}(z)=2|z-b| \log \frac{S}{|z-b|}
$$


par la projection:

$$
z=b+S \exp \left(\frac{w+1}{w-1}\right) \in \operatorname{Proj}(D, \Delta(b, S)) .
$$

Notre théorème 1 est, en effet, une conséquence immédiate du

THÉRÈME 1 BIS. Soit $a \in \partial G$ un point isolé de $\partial G$ et soit

Alors,

$$
R(a)=\inf \{|\zeta-a| ; \zeta \in \partial G \backslash\{a\}\} .
$$

$$
2|z-a| \log \frac{R(a)}{|z-a|} \leqq 1 / \Pi_{G}(z) \leqq 2|z-a| \log \frac{R(a)}{|z-a|}+2 c_{H}|z-a|
$$

pour $z \in\{z \in G ;|z-a| \leqq R(a)\}$, où

$$
c_{H}=\Gamma(1 / 4)^{4}\left(4 \pi^{2}\right)^{-1}=4.376 \cdots .
$$

L'égalité dans la première partie de (2.1) arrive en chaque $z \in G$ quand $G=$ $\Delta(a, S)$ car $R(a)=S$ dans ce cas. D'autre part, celle dans la seconde arrive en $z=-1$ dans le cas $G=C \backslash\{0,1\}$ et $a=0$. En effet, $2 I I_{C \backslash(0,1)}(-1)=1 / c_{H}$; voir [1, p. 436].

Posons $R=R(a)$ pour simplicité. Nous avons besoin du principe des métriques hyperboliques (ou, de Poincaré): Pour un sous-domaine $A$ d'un domaine hyperbolique $B$ nous avons:

$$
\Pi_{A}(z) \geqq \Pi_{B}(z) \quad \text { pour tout } z \in A ;
$$

voir $[3$, p. 50]. Alors, la première inégalité de (2.1) est une conséquence immédiate de ce principe par la relation $\Delta(a, R) \subset G$ avec la continuité de $\Pi_{G}$.

Il y a $q \in \partial G \backslash\{a\}$ tel que $|q-a|=R$. Alors, la relation $G \subset C \backslash\{a, q\}$ montre que

$$
1 / \Pi_{G}(z) \leqq 1 / \Pi_{C \backslash\{, q\}}(z) \quad \text { pour tout } z \in G .
$$

Puisque $z=(q-a) \zeta+a$ représente $C \backslash\{0,1\}$ sur $C \backslash\{a, q\}$, on a

$$
1 / \Pi_{C \backslash\{a, q\}}(z)=|q-a| / \Pi_{C \backslash\{0,1\}}\left(\frac{z-a}{q-a}\right)
$$

pour chaque $z \in C \backslash\{a, q\}$. D'autre part, J. A. Hempel a démontré l'inégalité du type de Landau en la forme exacte:

$$
1 / \Pi_{C \backslash\{0,1\}}(\zeta) \leqq 2|\zeta|\left(|\log | \zeta||+c_{H}\right)
$$

pour chaque $\zeta \in C \backslash\{0,1\}$; voir $[1,(4.1)]$. Notons que Hempel adopte $2 \Pi_{G}$ comme la densité de Poincaré. En combinant cette estimation avec (2.2) et (2.3) nous avons la seconde inégalité dans (2.1) par la continuité de $\Pi_{G}$. 
3. Allures de $\Pi_{G}$ près d'un $b \in \partial G$ général ou non isolé. En effet, (2.2) est vraie pour toute paire $a, q$ des points sur $\partial G$ pour autant que $a \neq q$ soit satisfaite; ici, $a$ n'est pas nécessairement isolé. Donc,

$$
1 / \Pi_{G}(z) \leqq 2|z-b|\left\{|\log | \frac{z-b}{q-b}||+c_{H}\right\}, \quad z \in G,
$$

où $b, q \in \partial G$ et $b \neq q$. Alors, on a

$$
\liminf _{z \rightarrow b} \Phi_{1}(z, b) \Pi_{G}(z) \geqq 1 / 2
$$

pour tout point $b \in \partial G$. En particulier, $\lim _{z \rightarrow b}|z-b|^{p} \Pi_{G}(z)=+\infty$ pour tout point $b \in \partial G$ et pour tout $0 \leqq p<1$.

Soit $b \in \partial G$. Supposons qu'il y a deux constantes $\varepsilon=\varepsilon(b)>0$ et $Q=Q(b) \geqq 1$ telles que, pour tout $r, 0<r<\varepsilon$, on puisse trouver $q=q(r) \in \partial G$ avec

$$
r / Q \leqq|q-b| \leqq r Q \text {. }
$$

Alors,

$$
1 / \Pi_{G}(z) \leqq 2\left(\log Q+c_{H}\right)|z-b| \text { pour } z \in G \text { avec }|z-b|<\varepsilon(b) .
$$

En effect, considérons (3.1) avec $b$ et $q=q(|z-b|)$. Évidemment, $b$ n'est pas isolé. En particulier, on a immédiatement,

$$
\liminf _{z \rightarrow b}|z-b| \Pi_{G}(z) \geqq 1 /\left(2 \log Q+2 c_{H}\right) .
$$

Deux tels exemples de $b$ sont proposés.

Example 1. Si la composante connexe de $\partial G$ contenant $b \in \partial G$ est un continu non dégénéré, alors on a $Q(b)=1$ toujours avec $\varepsilon(b)>0$ appropriée.

Example 2. Étant donné $Q>1$ nous posons $N$ le plus petit nombre naturel $\geqq 1 /\left(Q^{2}-1\right)$. Soit $P_{n}=Q / n$, et soit $G=C \backslash\left\{P_{n}\right\}_{n \geq N}$. Alors, pour $b=0 \in \partial G$ on a $\varepsilon=1 / N$ et $Q(b)=Q$. En effet, pour $r, 0<r<1 / N$, on a $n \geqq N$ tel que $1 /(n+1)$ $\leqq r<1 / n$. Ensuite, $r / Q<P_{n+1} \leqq r Q$, d'où $q=P_{n+1}$.

Il est facile d'observer que

$$
\liminf _{z \rightarrow 1}|z-1| \Pi_{D}(z)=1 / 2 .
$$

Est-ce que

$$
\limsup _{z \rightarrow b}|z-b| \Pi_{G}(z)<+\infty
$$

pour $b \in \partial G$ spécifié ci-dessus par $\varepsilon$ et $Q$ ? Un contre-exemple plus fort est pourvu par 


$$
\limsup _{z \rightarrow 1}|z-1|^{2} \Pi_{D}(z)=+\infty
$$

car $|z-1|^{2} \Pi_{D}(z)=1 / \operatorname{Re}\{(1+z) /(1-z)\}$. Encore, il y a un domaine $U$ tel que $U$ soit "maigre" près de $0 \in \partial U, \partial U$ étant un continu, et que

$$
\limsup _{z \rightarrow 0}|z|^{2} \Pi_{U}(z)=+\infty
$$

Le domaine cité est

$$
U=\left\{z ;\left|\operatorname{Im}\left(z^{-1}+2^{-1} \pi i\right)\right|<\pi / 2\right\}
$$

avec $\partial U=\left\{z ;|z|^{2}=\operatorname{Im} z / \pi\right.$ ou $\left.\operatorname{Im} z=0\right\}$ et 0 étant une cuspide de $\partial U$. Puisque

$$
1 / \Pi_{U}(z)=2|z|^{2} \sin \left(\frac{\operatorname{Im} z}{|z|^{2}}\right) \quad \text { pour } z \in U
$$

on a, sur un cercle $\left\{z ;|z|^{2}=(k / \pi) \operatorname{Im} z\right\}$ (pour tout $k>1$ fixé),

$$
|z|^{2} \Pi_{U}(z)=1 /(2 \sin (\pi / k)) \text {. }
$$

Maintenant, il est facile de démontrer (3.2). Notons que

$$
\liminf _{z \rightarrow 1}|z-1|^{2} \Pi_{D}(z)=0 \text { et toujours }|z|^{2} \Pi_{U}(z) \geqq 1 / 2 \text {. }
$$

4. Preuve du théorème 2. Pour $z \in G$ nous posons

$$
\delta_{G}(z)=\inf \{|\zeta-z| ; \zeta \in \partial G\} \quad \text { et } \quad \gamma_{G}(z)=\left|(\partial / \partial z)\left\{1 / \Pi_{G}(z)\right\}\right| \text {. }
$$

Nous connaissons l'inégalité exacte:

$$
\delta_{G}(z) \Pi_{G}(z) \leqq \frac{2}{2+\gamma_{G}(z)}, \quad z \in G ;
$$

voir $[4,(7.3)]$. Il résulte de (4.1) et de (2.1), avec $\delta_{G}(z)=|z-a|$ pour $0<|z-a|$ $\leqq R(a) / 2$, que

$$
\left|(\partial / \partial z) \Pi_{G}(z)\right| \leqq \frac{\log (R(a) /|z-a|)+c_{H}-1 / 2}{|z-a|^{2}(\log (R(a) /|z-a|))^{2}}
$$

pour $z \in\{0<|z-a| \leqq R(a) / 2\}$. Alors, il est facile de démontrer (1.3).

Après quelques calculs simples, partant de (1.1), on a

$$
\left(\partial^{2} / \partial z \partial \bar{z}\right) \Pi_{G}(z)=\Pi_{G}(z)^{3}+\left|(\partial / \partial z) \Pi_{G}(z)\right|^{2} \Pi_{G}(z)^{-1}, \quad z \in G,
$$

qui est essentiellement l'identité de la courbure de Gauss pour la métrique $\Pi_{G}(z)|d z|$, c'est-à-dire que

$$
\Delta \log \Pi_{G}(z)=4 \Pi_{G}(z)^{2}, \quad z \in G .
$$

Il résulte donc de $(2.1)$ et (4.2) que 


$$
\begin{aligned}
& \left(\partial^{2} / \partial z \partial \bar{z}\right) \Pi_{G}(z) \\
& \leqq \frac{2\left(\log (R(a) /|z-a|)+c_{H}\right)\left(\log (R(a) /|z-a|)+c_{H}-1 / 2\right)^{2}+(1 / 8) \log (R(a) /|z-a|)}{|z-a|^{3}(\log (R(a) /|z-a|))^{4}}
\end{aligned}
$$

pour $z \in\{0<|z-a| \leqq R(a) / 2\}$. L'inégalité (1.5) alors s'obtient immédiatement.

Pour dèmontrer (1.4) nous allons utiliser l'inégalité exacte :

$$
\left(1-|w|^{2}\right)^{2}\left|\frac{g^{\prime \prime \prime}(w)}{g^{\prime}(w)}-\frac{3}{2}\left(\frac{g^{\prime \prime}(w)}{g^{\prime}(w)}\right)^{2}\right| \leqq 6\left(\left\{\frac{\left(1-|w|^{2}\right)\left|g^{\prime}(w)\right|}{\delta(w, g)}\right\}^{2}-1\right)
$$

pour $w \in D$ où $g$ est holomorphe dans $D, g^{\prime}(w) \neq 0$, et $\delta(w, g)$ est le plus grand $r>0$ tel que le disque d'une feuille et du centre $g(w),\{\zeta ;|\zeta-g(w)|<r\}$, se situe sur la surface de Riemann qui est l'image de $D$ par $g$; voir $[4,(6.2)]$.

Puisque nous avons, pour $f \in \operatorname{Proj}(D, G)$, que $\delta_{G}(z)=\delta(w, f), z=f(w) \in G$, et, de plus, un calcul montre que

$$
\left\{2 / \Pi_{G}(z)\right\}\left|\left(\partial^{2} / \partial z^{2}\right)\left\{1 / \Pi_{G}(z)\right\}\right|=\left(1-|w|^{2}\right)^{2}\left|\frac{f^{\prime \prime \prime}(w)}{f^{\prime}(w)}-\frac{3}{2}\left(\frac{f^{\prime \prime}(w)}{f^{\prime}(w)}\right)^{2}\right|,
$$

on a, avec l'aide de (4.3) pour $g=f$, que

$$
\left|\left(\partial^{2} / \partial z^{2}\right) \Pi_{G}(z)-2 \Pi_{G}(z)^{-1}\left\{(\partial / \partial z) \Pi_{G}(z)\right\}^{2}\right| \leqq 3 \Pi_{G}(z)^{3}\left(\left\{\delta_{G}(z) \Pi_{G}(z)\right\}^{-2}-1\right), \quad z \in G .
$$

Il résulte donc de (2.1) et (4.2) que

$$
\begin{aligned}
& \left|\left(\partial^{2} / \partial z^{2}\right) \Pi_{G}(z)\right| \\
& \leqq \frac{4\left(\log (R(a) /|z-a|)+c_{H}\right)\left(\log (R(a) /|z-a|)+c_{H}-1 / 2\right)^{2}+(3 / 2)(\log (R(a) /|z-a|))^{3}}{|z-a|^{3}(\log R(a) /|z-a|)^{4}} \\
& -\frac{3}{8|z-a|^{3}\left(\log (R(a) /|z-a|)+c_{H}\right)^{3}}
\end{aligned}
$$

pour $z \in\{0<|z-a| \leqq R(a) / 2\}$. Cette estimation dèmontre (1.4).

Il est facile de calculer les suivantes pour $z \in H$,

$$
\begin{gathered}
(\partial / \partial z) \Pi_{H}(z)=\frac{\log |z|+1}{4 z|z|(\log |z|)^{2}}, \\
\left(\partial^{2} / \partial z^{2}\right) \Pi_{H}(z)=\frac{3(\log |z|)^{2}+4 \log |z|+2}{-8 z^{2}|z|(\log |z|)^{3}}, \\
\left(\partial^{2} / \partial z \partial \bar{z}\right) \Pi_{H}(z)=\frac{(\log |z|)^{2}+2 \log |z|+2}{-8|z|^{3}(\log |z|)^{3}},
\end{gathered}
$$

et ces identités montrent (1.6)-(1.8). 
Remarque 4.1. Si la dérivée $g^{\prime}$ de $g$ holomorphe dans $D$ ne s'annule jamais dans $D$ et si

$$
\left(1-|w|^{2}\right)\left|g^{\prime}(w)\right| \leqq(2 / \sqrt{3}) \delta(w, g) \text { pour tout } w \in D,
$$

alors, $g$ est univalente dans $D$. En effect, dans ce cas, on a

$$
\sup _{w \in D}\left(1-|w|^{2}\right)^{2}\left|\frac{g^{\prime \prime \prime}(w)}{g^{\prime}(w)}-\frac{3}{2}\left(\frac{g^{\prime \prime}(w)}{g^{\prime}(w)}\right)^{2}\right| \leqq 2
$$

par (4.3). Le théorème bien connu de Z. Nehari [2] démontre l'univalence de l'univalence $g$ dans $D$.

5. Le rayon d'univalence. Pour $f \in \operatorname{Proj}(D, G)$ et $w \in D$, nous désignons par $\rho(w, f)$ le maximum de $r, 0<r \leqq 1$, tel que $f$ soit univalente dans un disque d'Apollonius $\{\zeta ;|\zeta-w| /|1-\bar{w} \zeta|<r\}$. Nous définissons $\rho_{G}(z), z \in G$, par $\rho_{G}(z)$ $=\rho(w, f), z=f(w)$. Donc, $\rho_{G}(z)$ est bien définie parce que $\rho(w, f)=\rho\left(w^{\prime}, f\right)$ si $f(w)=f\left(w^{\prime}\right)$. De plus, $\rho_{G}(z)$ est indépendante du choix particulier de $f$. La quantité $\rho_{G}(z)$ s'appelle le rayon d'univalence de $G$ en $z \in G$. On connait l'estimation exacte pour $g$ holomorphe et univalente dans $G$ :

$$
\left\{\rho_{G}(z) / \Pi_{G}(z)\right\}\left|g^{\prime \prime}(z) / g^{\prime}(z)\right| \leqq 8 \quad \text { pour tout } z \in G ;
$$

voir [5].

Il y a des étroites relations entre $\rho_{G}$ et $\Pi_{G}$; par suite, nous pouvons étudier aussi l'allure de $\rho_{G}(z)$ au voisinage de $a \in \partial G$ isolé. Notre résultat est:

THÉORÈme 3. Soıt $a \in \partial G$ un point isolé de $\partial G$. Alors, pour le rayon d'univalence $\rho_{G}$ nous avons

$$
1 / 8 \leqq \liminf _{z \rightarrow a}\left(\log \frac{1}{|z-a|}\right) \rho_{G}(z) \text { et } \limsup _{z \rightarrow a}\left(\log \frac{1}{|z-a|}\right) \rho_{G}(z) \leqq 2 .
$$

Par $\rho_{G}(z) \gamma_{G}(z) \leqq 2$ et $\rho_{G}(z) \leqq\left\{2+\rho_{G}(z) \gamma_{G}(z)\right\} \delta_{G}(z) \Pi_{G}(z)$ pour $z \in G(\operatorname{voir}[4,(7.1)$ et $(7.2)]$ ) on obtienne

$$
\rho_{G}(z) \leqq 4 \delta_{G}(z) \Pi_{G}(z) .
$$

Donc, nous avons l'estimation:

$$
\left(\log \frac{1}{|z-a|}\right) \rho_{G}(z) \leqq 4 \Phi_{1}(z, a) \Pi_{G}(z)
$$

pour $0<|z-a| \leqq R(a) / 2$. La seconde partie de (5.2) est alors une consequence de (1.2). 
Par

$$
\delta_{G}(z) \Pi_{G}(z)\left(\rho_{G}(z)+1\right)^{2} \leqq 4 \rho_{G}(z), \quad z \in G
$$

(voir $[4,(7.4)])$ on a $\delta_{G}(z) \Pi_{G}(z) \leqq 4 \rho_{G}(z)$. Donc,

$$
\Phi_{1}(z, a) \Pi_{G}(z) \leqq 4\left(\log \frac{1}{|z-a|}\right) \rho_{G}(z)
$$

pour $0<|z-a| \leqq R(a) / 2$, d'où on a la première partie de (5.2) par (1.2).

Remarqne 5.1. Nous pouvons obtenir la seconde inégalité dans (5.2) aussi par (5.1) en posant $g(z)=1 /(z-a)$. En combinant (1.2) avec $|z-a|\left|g^{\prime \prime}(z) / g^{\prime}(z)\right|$ $\equiv 2$ on a l'inégalité.

Remarque 5.2. On peut démontrer

$$
\rho_{H}(z)=\pi^{-1}\left(\log |z|+\left\{(\log |z|)^{2}+\pi^{2}\right\}^{1 / 2}\right), \quad z \in H .
$$

En effect, par $\eta=\varphi(\zeta)=(\zeta+1) /(\zeta-1)$, le disque d'Apollonius $\{\zeta ;|\zeta-w| / \mid 1-$ $\bar{w} \zeta \mid<r\}(w \in D, 0<r \leqq 1)$ dans $D$ est représenté sur le disque d'Apollonius $\{\eta$; $|\eta-\varphi(w)| /|\eta+\overline{\varphi(w)}|<r\}$ dans le demi-plan gauche, dont le diamètre euclidien est $E(r)=-4 r \operatorname{Re} \varphi(w) /\left(1-r^{2}\right)$. En résolvant l'équation de $r: E(r)=2 \pi$ pour $\operatorname{Re} \varphi(w)=\log |z|(z \in H)$ donnée, on a $r=\rho_{H}(z)$.

Finalemant il est facile de déduire

$$
\lim _{z \rightarrow 0}(-\log |z|) \rho_{H}(z)=\pi / 2 .
$$

Donc, une conjecture un peu hardie est que:

$$
\lim _{z \rightarrow a}\left(\log \frac{1}{|z-a|}\right) \rho_{G}(z)=\pi / 2
$$

pour tout point $a \in \partial G$ isolé.

\section{Références bibliographiques}

[1] J.A. Hempel, The Poincaré metric on the twice punctured plane and the theorems of Landau and Schottky. J. London Math. Soc. 20 (1979), 435-445.

[2] Z. NEHARI, The Schwarzian derivative and schlicht functions. Bull. Amer. Math. Soc. 55 (1949), 545-551.

[3] R. Nevanlinna, Eindeutige analytische Funktionen. Springer-Verlag, BerlinHeidelberg-New York, 1974.

[4] S. Yamashita, The derivative of a holomorphic function and estimates of the Poincaré density. Kodai Math. J. 15 (1992), 102-121.

[5] S. Yamashita, La dérivée d'une fonction univalente dans un domaine hyperbolique. C. R. Acad. Sci. Paris 314 (1992), 45-48.

[6] A. YAMADA, Bounded analytic functions and metrics of constant curvature on Riemann surfaces. Kodai Math. J. 11 (1988), 317-324. 
ALLURES DE LA DENSITÉ DE POINCARÉ ET SES DÉRIVÉES

Département de Mathématique,

UNIVERSITE MÉTROPOLITAINE DE TOKYO,

Minami-Osawa, Hachioji, Tokyo 192-03

JAPON 